(D)

CORPUS PUBLISHERS

\section{Current Research} in Emergency Medicine (CREM)

Volume 2, Issue 1, 2022

Article Information

Received date : 11 January, 2022

Published date: 20 January, 2022

\section{*Corresponding author}

John DeAngelis, MD, RDMS, FACEP, 601 Elmwood Ave Box 655, Rochester NY 14642, 585-270-1232

\section{Key Words}

POCUS; Endocarditis; Ultrasound;

Diagnosis; Emergency Department

Distributed under: Creative Commons CC-BY 4.0

\title{
Endocarditis in the Emergency Department: A POCUS Case Series
}

\author{
Weaam Alshenawy ${ }^{1}$, Hussain Alrobeh ${ }^{2}$, Carl Mirus IV ${ }^{3}$, John DeAngelis ${ }^{4^{*}}$ \\ ${ }^{1}$ Weaam Alshenawy, MBBS, Emergency Medicine Associate Consultant, King Faisal Specialists Hospital. \\ Riyadh, Saudi Arabia \\ ${ }^{2}$ Hussain Alrobeh, MBBS, King Faisal Specialists Hospital. Riyadh, Saudi Arabia
}

${ }^{3}$ Carl Mirus IV, MD, Assistant Professor of Emergency Medicine, UT Southwestern Medical Center, Dallas, $T X$

${ }^{4}$ John DeAngelis, MD, Assistant Professor of Emergency Medicine,University of Rochester Medical Center, Rochester, New York

\section{Abstract}

\section{Introduction}

Infectious endocarditis remains a difficult to diagnose entity in the emergency department. This is partially due to vague and non-specific presenting symptoms but also because the Major Duke criteria $[1,2]$ are difficult to achieve in the timeframe of the standard emergency department visit. This can lead to diagnostic uncertainty and delay in treatment. Point of Care Ultrasound (POCUS) has grown significantly in the emergency setting and is now readily available in most departments [3]. Utilizing POCUS in this difficult subset of patients can successfully identify endocarditis earlier and lead to improved treatment times.

\section{Cases}

Each of the three cases in this report demonstrate an episode of care in which the patient presented with non-specific symptoms. In two, endocarditis was suspected, while in the third case there was significant distraction to make the diagnosis very difficult. In each case, POCUS was applied successfully confirming the diagnosis of endocarditis when added to the minor Duke criteria already present. This led to a confirmation of diagnosis up to 12 hours before formal confirmation during the patients' admissions.

\section{Conclusion}

While this specific indication for POCUS is currently outside the core utilization in the ED, and significant limitations remain: i.e. unknown sensitivity, this series demonstrates that POCUS provides value in the work up of suspected endocarditis by confirming the diagnosis earlier. This series suggests that further research into the sensitivity of POCUS for endocarditis in the ED may be warranted.

\section{Introduction}

Infectious endocarditis is a rare, difficult to diagnose disorder with vague and non-specific symptoms until late in the natural history of the disease. Early diagnosis of infective endocarditis is crucial because of high mortality rates $[4,5]$ and although prompt identification and surgical intervention has reduced its mortality rate [4,6], the diagnosis continues to be challenging in the Emergency Department (ED). The importance of rapid diagnosis is highlighted by Benedetto et al who noted that "some of the available evidences showed that are no proven benefits in delaying surgery if a definite diagnosis of [infective endocarditis] has been established [7]." The main diagnostic criteria, the Duke Criteria [1,2], rely on formal ultrasonography, blood cultures, or late stage physical exam findings to make the diagnosis. The two major criteria are not immediately available, which, when combined with the psychosocial difficulties of the injection drug user population, leads to likely many missed early cases of endocarditis. In this series we demonstrate 3 cases where Point-of-Care Ultrasound (POCUS) was utilized in the ED to diagnosis endocarditis in a time sensitive way by visualizing valvular vegetations. This allowed for early antibiotic administration and a life-threatening diagnosis to be made up to 12 hours before these cases were confirmed by positive blood cultures and formal ultrasonography. Time which could be used to stabilize and get patients to surgery sooner for definitive treatment.

\section{Case 1}

This is a case of a 30-year-old Caucasian male with a history of polysubstance abuse, including Injection Drug Use (IDU) with heroin. He reported a one-week history of feeling generally unwell with flu-like symptoms that were associated with intermittent cough, chest pain, arthralgia, and myalgia. Initially, his mother took him to urgent care as his symptoms worsened and he was found to have a fever of $39.4 \mathrm{C}$. He was diagnosed with the flu and sent home. However, the patient became more lethargic and confused the next day, which prompted his mother to bring him to the ED. In the ED, he was initially afebrile at 37.4 C, HR 123, BP 160/88, RR 22, and O2sat 96\%. On exam, he was confused with no apparent neurological deficit but he was unable to provide a cogent history. His cardiac exam was significant for an apical systolic murmur of II/VI radiating to the left axilla. His labs were notable for leukocytosis of 13,200 with left shift, and severe anemia with $\mathrm{Hgb} / \mathrm{Hct}$ of 5.2/17 respectively. Urine toxicology screening was positive for cocaine, THC, and benzodiazepine but not opiates. CT chest showed multiple cavitary lesions consistent with septic emboli. He met 3 minor Duke Criteria in the Emergency Department to this point. Bedside POCUS was obtained due to high suspicion of endocarditis and demonstrated a Tricuspid valve mass compatible with vegetation (Figure 1) which provided enough information to diagnosis him with acute endocarditis and start therapy. This was confirmed with two sets of blood cultures that grew methicillin-susceptible Staphylococcus aureus (with time to positivity of $\sim 11$ hours), as well as a conventional Transthoracic Echocardiogram (TTE) a day later that confirmed a mobile tricuspid valve mass of $1.5 \times 1 \mathrm{~cm}$ with mild TV insufficiency. 


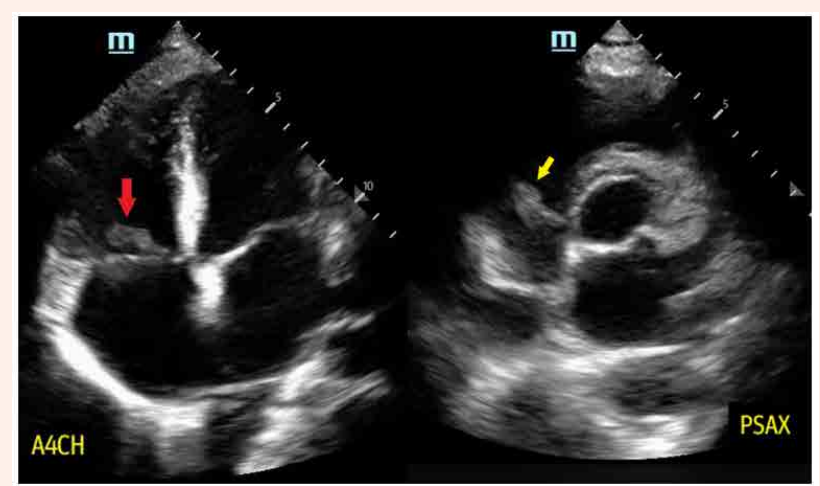

Figure 1: Tricuspid vegetation seen in both A4 and PSAX views indicated by red and yellow arrows respectively

\section{Case 2}

This is a case of a 35-year-old female with a medical history of IDU who presented to our emergency department as a transfer from an outside community hospital for concern of septic pulmonary emboli and altered mental status. A week prior, the patient presented to an outside hospital with chest pain, fever, and cough. Imaging was consistent with multiple septic pulmonary emboli. The patient left against medical advice and returned a few days later, presenting to our emergency department confused, febrile, tachypneic, and tachycardic. The patient appeared ill, and disoriented. She was afebrile at 36.5 C, HR 116, BP 108/53, RR 18, and O2Sat 96\%. Auscultation was positive for an apical systolic murmur radiating to the left axilla. Labs were significant for leukocytosis 17,300 with left shift and mild anemia, slightly elevated liver enzymes and lactate of 2.4. A chest X-ray showed multiple cavitary lesions that were confirmed by CT chest. Per the modified Duke criteria, she had 3 minor criteria (fever, septic emboli and a risk factor of IDU). Given that, the Emergency Medicine team performed a cardiac POCUS to assess for valvular vegetations. A tricuspid valve vegetation was discovered (Figure 2) which in turn increased the likelihood of endocarditis from probable to definite according to the Duke Criteria (1 major echocardiographi evidence of vegetation and 3 minor). Two sets of blood cultures subsequently returned positive for MSSA (with time to positivity of $\sim 12$ hours). A formal TTE was significant for tricuspid regurgitation with vegetation.

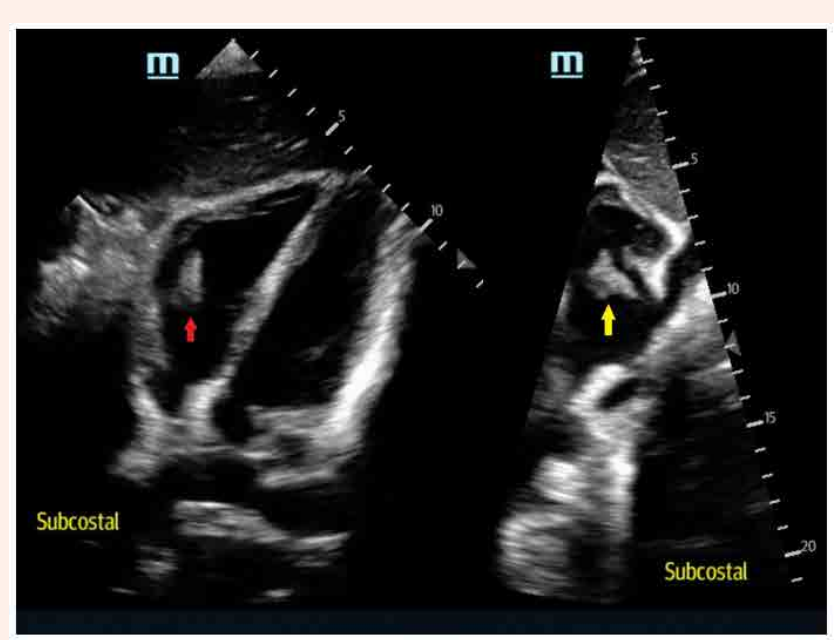

Figure 2: Tricuspid vegetation visualized in the subcostal view (red arrow) and in a modified subcostal visualizing the IVC (yellow arrow)

Case 3

This is a case of a 41-year-old female with past medical history of Buerger's disease, Raynaud's disease and history of opioid dependence on opioid replacement therapy (denying any recent IDU) presenting to the Emergency Department with intermittent word-finding difficulty, headache and left-handed weakness. She was diagnosed four months prior with splenomegaly, a splenic laceration, and enlarged axillary lymph nodes in the setting of anemia that was concerning for a lymphoproliferative disorder. The patient had also been experiencing nightly fevers and diaphoresis which started around the same time. She was seen outpatient and referred to Hematology/Oncology for lymph node biopsy and further work-up. Before further work-up occurred, she presented to an outside Emergency Department and was found to have a hemoglobin below 15 and subsequently transferred. On arrival to our Emergency Department, the patient was oriented, anxious, tachypneic, and slow to respond to questioning without focal neurological deficits. Vitals on arrival were 39C, HR 117, BP 120/64, RR 22, O2sat $94 \%$ on $3 \mathrm{~L}$ nasal cannula. Labs were remarkable for pancytopenia with a $\mathrm{H} / \mathrm{H} 4.4 / 14$, WBC $2.7 \mathrm{k}$, Platelets $99 \mathrm{k}$, Cr of $6.51 \mathrm{mg} / \mathrm{dl}$, BUN $64 \mathrm{mg} / \mathrm{dl}$, and BNP $14,985 \mathrm{pg} / \mathrm{mL}$. CT imaging of the head showed an ill-defined lesion in the right frontal lobe that was nonspecific, interpreted as a subacute infarct versus chronic small vessel disease versus calcification. CT of the chest showed pulmonary edema with enlarged mediastinal lymph nodes.

The patient was in multi-organ failure secondary to poor perfusion from severe anemia thought to be from a blood dyscrasia versus an oozing splenic laceration. The patient responded well to a blood transfusion, vital signs stabilized and there continued to be no neurological deficit on exam. While in the ED, she became hypotensive and had worsening encephalopathy, which did not correlate with a diagnosis of a lymphoproliferative disorder. The patient met 3 minor Duke Criteria: fever, evidence of embolic phenomena, and evidence of infection by her lymphadenopathy. She also had a distant history of opiate abuse, though she currently denied active utilization.

At this time, a cardiac POCUS was obtained to assess for cardiac function in the setting of new hypotension of unclear etiology. This demonstrated a concerning mitral valve lesion (Figure 3). Infective endocarditis, which was initially felt to be less likely, became our immediate main focus as she now had 1 major and 3 minor criteria. Based off of the findings of the cardiac POCUS, there was quick change in therapy to antibiotics and further management for sepsis and septic emboli. Blood cultures subsequently grew gram-positive cocci $\sim 12$ hours later and transesophageal echocardiogram the next day during inpatient workup was significant for mitral and aortic valve masses.

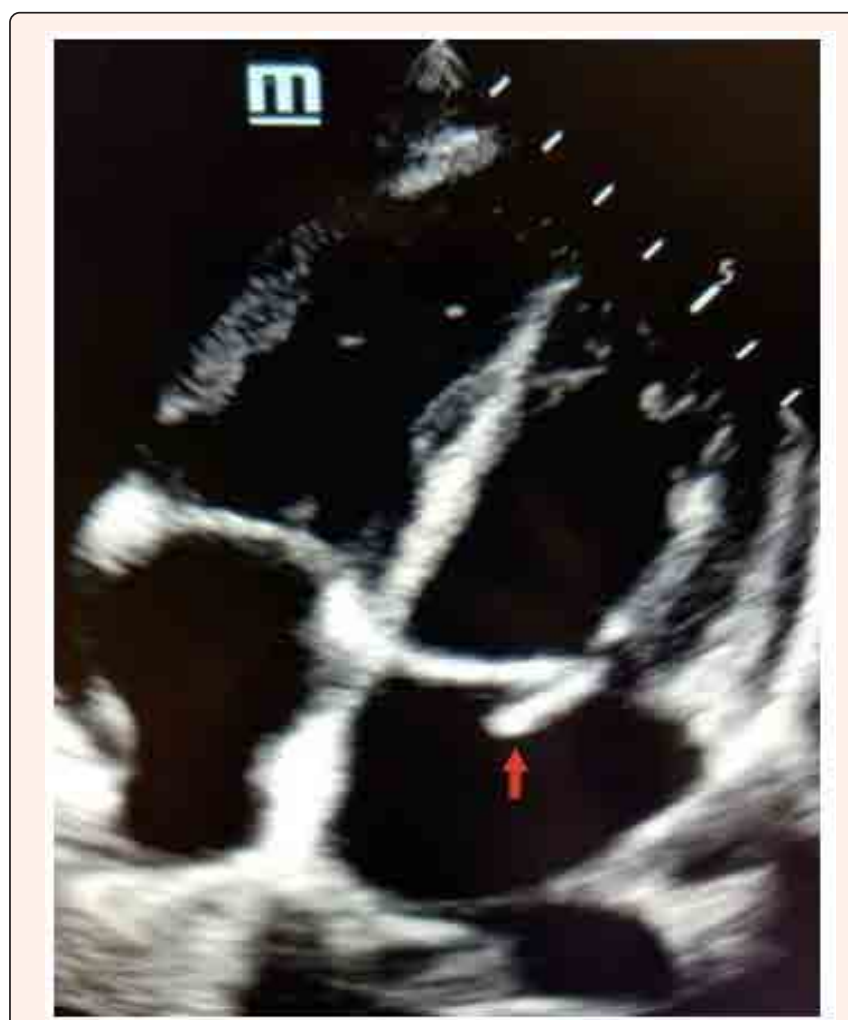

Figure 3: Mitral valve vegetation seen on the A4 view (red arrow)

Citation: Alshenawy W, Alrobeh H, Mirus IV C, DeAngelis J (2022) Endocarditis in the Emergency Department: A POCUS Case Series. Curr Res Emerg Med 2: 1017 


\section{Discussion}

Based on the Duke Criteria, these patients would not have met the diagnosis of endocarditis upon initial presentation and resuscitation in the emergency department. Although the role of POCUS in diagnosing endocarditis has not yet been well established, there has been an increasing amount of case reports where POCUS evidence of valvular lesions has changed the course of management [8-12]. Though it is important to note that the absence of valvular lesions does not rule out the possibility of infective endocarditis. In our case, it shifted the management immediately, and in Case 3 specifically prevented pursuit of an incorrect diagnosis of lymphoproliferative disease. In each of these cases the diagnosis of endocarditis was confirmed approximately 12 hours earlier than would have been expected, had POCUS not been utilized. Guidelines in the treatment of endocarditis suggest that prompt and early administration of IV antibiotics is key to the treatment of this disease [4], and since sepsis is often present at presentation early antibiotics in the first hour is recommended [13]. Through the utilization of POCUS in the ED we were able to confirm this diagnosis and improve the efficiency and rapidity of treatment.

Table 1: Diagnosis of endocarditis requires: 2 major, 1 major and 3 minor, or 5 minor criteria

\begin{tabular}{|c|c|}
\hline & Modified Duke Criteria for the Diagnosis of Endocarditis \\
\hline \multirow[b]{2}{*}{$\begin{array}{l}\text { Major } \\
\text { Criteria }\end{array}$} & Two separate positive blood cultures \\
\hline & $\begin{array}{l}\text { Echocardiographic evidence of valvular vegetations, abscess, or new } \\
\text { regurgitation }\end{array}$ \\
\hline \multirow{5}{*}{$\begin{array}{l}\text { Minor } \\
\text { Criteria }\end{array}$} & Predisposition (IDU, or other risk) \\
\hline & Temperature $>38 \mathrm{C}$ \\
\hline & $\begin{array}{l}\text { Vascular phenomena: Emboli, infarcts, mycotic aneurysm, stroke, } \\
\text { conjunctival hemorrhage, Janeway lesions, splinter hemorrhages }\end{array}$ \\
\hline & Immunologic phenomena: Osler nodes, Roth spots, glomerulonephritis \\
\hline & $\begin{array}{l}\text { Microbiologic evidence: serologic evidence of active infection or positive } \\
\text { blood culture not meeting major criteria }\end{array}$ \\
\hline
\end{tabular}

\section{Conclusion}

While these cases should not be construed to suggest that valvular vegetation evaluation in the Emergency Department should become a core aspect of cardiac POCUS, it demonstrates that POCUS can pick up extra information that can be clinically relevant beyond the core applications and that recognition by the advanced POCUS provider can change clinical management and improve patient care. As familiarity, skill, and availability of POCUS in the Emergency Department continues to grow, practitioners should continue to expand what is possible with an ultrasound probe, and this series suggests that further investigation of cardiac POCUS for the evaluation of endocarditis in the Emergency Department may be warranted.

\section{References}

1. Durack DT, Lukes AS, Bright DK (1994) New criteria for diagnosis of infective endocarditis: utilization of specific echocardiographic findings. Duke Endocarditis Service. Am J Med 96(3): 200-209.

2. Li JS, Sexton DJ, Mick N, Nettles R, Fowler VG, et al. (2000) Proposed modifications to the Duke criteria for the diagnosis of infective endocarditis. Clin Infect Dis 30(4): 633-638.

3. Bobbia X, Abou-Badra M, Hansel N, Pes P, Petrovic T, et al. (2018) Changes in the availability of bedside ultrasound practice in emergency rooms and prehospital settings in France. Anaesth Crit Care Pain Med 37(3): 201-205.

4. Cahill TJ, Prendergast BD (2016) Infective endocarditis. Lancet 387(10021):882893.

5. Sunder S, Guillon LG, Lemaignen A, Lacasse M, Gaborit C, et al. (2019) Incidence, characteristics, and mortality of infective endocarditis in France in 2011. PLoS One 14(10): e0223857.

6. Vincent LL, Otto CM (2018) Infective Endocarditis: Update on Epidemiology, Outcomes, and Management. Curr Cardiol Rep 20(10): 86.

7. Benedetto U, Spadaccio C, Gentile F, Moon MR, Nappi F (2020) A narrative review of early surgery versus conventional treatment for infective endocarditis: do we have an answer? Ann Transl Med 8(23): 1626.

8. Arthur J, Havyarimana J, Norse AB (2018) Emergency Department Diagnosis of Septic Pulmonary Embolism due to Infectious Endocarditis Using Bedside Ultrasound. J Emerg Med 55(3): 378-382.

9. Cohen A, Greco J, Levitus M, Nelson M (2020) The use of point-of-care ultrasound to diagnose infective endocarditis causing an NSTEMI in a patient with chest pain. J Am Coll Emerg Physicians Open 1(2): 120-123.

10. Fischer BG, Baduashvili A (2019) Cardiac Point-of-Care Ultrasound for the Diagnosis of Infective Endocarditis in a Patient with Non-Specific Rheumatologic Symptoms and Glomerulonephritis. Am J Case Rep 20: 542-547.

11. Seif D, Meeks A, Mailhot T, Perera P (2013) Emergency department diagnosis of infective endocarditis using bedside emergency ultrasound. Crit Ultrasound J 5(1): 1 .

12. Taylor J, Braver O, Fuchs L (2017) Infective Endocarditis Diagnosed by Pocket Ultrasound at the Bedside. J Ultrasound Med 36(11): 2392- 2394.

13. De Backer D, Dorman T (2017) Surviving sepsis guidelines: A continuous move toward better care of patients with sepsis. JAMA 317(8): 807-808. 